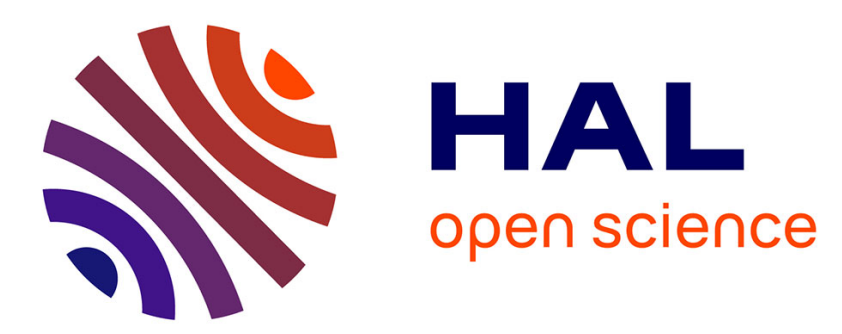

\title{
Two-liquid wetting properties as a surface polarity probe for hydrophobic coatings
}

\author{
G. Bonfante, S. Chevalliot, B. Toury, B. Berge, Mathieu Maillard
}

\section{To cite this version:}

G. Bonfante, S. Chevalliot, B. Toury, B. Berge, Mathieu Maillard. Two-liquid wetting properties as a surface polarity probe for hydrophobic coatings. Physical Chemistry Chemical Physics, 2017, 19 (4), pp.3214-3218. 10.1039/C6CP07392A . hal-01595951

\section{HAL Id: hal-01595951 \\ https://hal.science/hal-01595951}

Submitted on 13 Apr 2021

HAL is a multi-disciplinary open access archive for the deposit and dissemination of scientific research documents, whether they are published or not. The documents may come from teaching and research institutions in France or abroad, or from public or private research centers.
L'archive ouverte pluridisciplinaire HAL, est destinée au dépôt et à la diffusion de documents scientifiques de niveau recherche, publiés ou non, émanant des établissements d'enseignement et de recherche français ou étrangers, des laboratoires publics ou privés. 


\title{
Two liquids wetting properties as a surface polarity probe for hydrophobic coatings
}

\author{
G. Bonfante ${ }^{\mathrm{ab}}$, S. Chevalliot ${ }^{\mathrm{a}}$, B. Toury ${ }^{\mathrm{b}}$, B. Berge $^{\mathrm{a}}$, M. Maillard $^{\mathrm{a}}$
}

A model describing a non-polar liquid spreading on a surface within a polar medium is described theoretically, according to Fowkes Good and Girifalco approximations on interfacial tension. We demonstrate both theoretically and experimentally that surface polarity measurements using contact angle from two immiscible liquids minimize drastically the measurement error. The present method has been successfully applied to various substrates of variable polarity and overall surface energy. We also demonstrate that this method allows a direct measurement of surface sensitivity to $\mathrm{pH}$.

a Varioptic, Parrot SA Ba^timent Tony Garnier, 24 rue Jean Baldassini, 69007 Lyon, France. E-mail: mathieu.maillard@univ-lyon1.fr

b Universite' de Lyon, Laboratoire des Multimate'riaux et Interfaces, UMR 5615, CNRS-Universite' Lyon 143 bd du 11 Novembre 1918 , F-69622 Villeurbanne,

France

\section{Introduction}

Surface energy and more specifically introduction of polar and apolar components by Fowkes ${ }^{1,2}$ in the $50^{\prime}$ s have been a breakthrough in the understanding of not only liquid wettability but also more complex systems implying a competition of spreading or adhesion of two or more compounds ${ }^{3}$ on a surface due to a hydrophilic - lipophilic balance between compounds. A fairly large scientific and technologic domain concerning non adhesive coatings relies on reliability of non-polar coatings like Micro Electro Mechanical Systems (MEMS) or optical coatings. Adhesion of proteins and living cells ${ }^{4}$ in organisms are also related to adhesion in a surrounding medium ${ }^{5-7}$. This has been made possible by an extended theoretical work from the Young's equation ${ }^{8}$ in the early $19^{\text {th }}$ century to more recently the establishment of the method ${ }^{9,10}$ from Owens, Wendt, Rabel and Kaelble (OWRK), measuring contact angle from at least two liquids of known surface tension, and having a large chemical difference in terms of polarity. This method is, now, widely used and very well adapted for most surfaces. It is based on the contact angle measurement of one liquid surrounded by its vapor, but consequently it does not mimic exactly the situation where adhesion or spreading from one element occurs in a condensed medium. In this case, spreading is a competition between two compounds chemical affinity with a surface. Also, measuring contact angle from a non-polar liquid in absence of water, does not take into account specific interactions of aqueous or polar medium with the substrate of interest. In particular, it cannot take into account that surface somehow reacts with one of the liquid phase, by $\mathrm{pH}$-dependent proton exchange, surfactant adsorption or material swelling for instance ${ }^{11}$. Some alternative method ${ }^{2}$ using contact angle hysteresis ${ }^{12}$ and electron donicity ${ }^{13}$ have been developed, in particular for biological interactions, but are also limited to low surface energy since they imply only one liquid spreading at a time, on a surface.

\section{Theory}

The most standard method for determination of surface energy and surface polarity is based on Owens, Wendt, Rabel and Kaelble (OWRK) method $^{9,10}$. This method relies on Young's equation combined with Owen, Good and Girifalco approximation. Young's equation describes contact angle $\theta_{l}$ from a drop of liquid $l$ at equilibrium of interfacial forces at the triple line between a supporting solid, the liquid and its vapor:

$$
\gamma_{s}=\gamma_{s l}+\gamma_{l} \cos \theta_{l}(1)
$$


with $\gamma_{s}, \gamma_{s l}$ and $\gamma_{l}$ the surface energy, solid-liquid interface tension and liquid-vapor surface tension. Owen, Good and Girifalco approximation is based on dispersive and polar interactions, and describing interfacial tension as a function of individual surface tensions:

$$
\gamma_{s l}=\gamma_{s}+\gamma_{l}-2 \sqrt{\gamma_{s}^{d} \gamma_{l}^{d}}-2 \sqrt{\gamma_{s}^{p} \gamma_{l}^{p}}
$$

With $\gamma_{l}^{p}$ and $\gamma_{l}^{d}$ corresponding to polar and dispersive components from liquid surface tension and $\gamma_{s}^{p}$ and $\gamma_{s}^{d}$ corresponding to polar and dispersive components from surface energy. Combining equations 1 and 2 leads to a linearized equation as a function of both polar and dispersive components from surface energies:

$$
\frac{\gamma_{l}}{2 \sqrt{\gamma_{l}^{d}}}\left(1+\cos \theta_{l}\right)=\sqrt{\gamma_{s}^{d}}+\sqrt{\gamma_{s}^{p}} \frac{\sqrt{\gamma_{l}^{p}}}{\sqrt{\gamma_{l}^{d}}}=X \times \sqrt{\gamma_{s}^{p}}+\sqrt{\gamma_{s}^{d}}
$$

leading to a linear equation $\mathrm{Y}=\mathrm{AX}+\mathrm{B}$ with $X=\frac{\sqrt{\gamma_{l}^{p}}}{\sqrt{\gamma_{l}^{d}}}$ and $Y=\frac{\gamma_{l}}{2 \sqrt{\gamma_{l}^{d}}}\left(1+\cos \theta_{l}\right)$.

In this equation the square root of polar and dispersive surface energy components correspond to the slope and y-intercept of the affine function, respectively.

Increasing the number of measurements, further improves accuracy of polarity measurements but substrates can be sensitive to probing liquids. In addition, it is sometimes required to use a fresh sample for each measurement because a previous liquid deposition can influence the next measurement, which makes it more complex in terms of sample reproducibility. Increasing the required number of measurements obviously makes it also more difficult for a routine test in industrial applications. This method is also limited to low surface energies, since liquids having a lower surface tension than supporting substrates are in a total spreading situation according to Zisman law $^{14}$, which of course prevents from any surface polarity measurement on high surface energy substrates.

\section{Experiments}

One and two liquids contact angle measurements have been performed on a Kruss easydrop ${ }^{\circledR}$ goniometer and contact angle has been measured with the associated software Drop Shape Analysis using water ${ }^{15}$, decane ${ }^{16}$, octane ${ }^{16}$, diodomethane ${ }^{17}$, ethylene glycol ${ }^{18,19}$, and bromonaphtalene $^{18}$ as reference liquids. Aqueous solutions of variable $\mathrm{pH}$ have been obtained diluting hydrochloric acid and sodium hydroxide in ultra-pure water. In the present work, we used Parylene $\mathrm{C}$ coatings ${ }^{20}$ and SiOC, covered or not with fluorinated coatings of Cytop and fluoropel as substrates (see details in ESI S1).

Each surface is tested with at least four different liquids. For each tested liquid, three drops of $3 \mu \mathrm{L}$ are deposited onto the surface, measuring the advancing contact angle in an ambient medium. Since substrates are made of $<100>$ silicon wafer, surface roughness is extremely low $\left(r_{a}<1 n m\right)$ and deposition methods for Parylene $C$ and top-coats do not significantly increase roughness ${ }^{21,22}$.

\section{Results and discussion}

Based on Fowkes, Good and Girifalco approximations and previously published results ${ }^{14}$ we develop a predictive model for surface polarity based on two liquids wetting: assuming an oil drop medium (o) surrounded by a polar and immiscible medium (w) standing on a flat surface (s) we can write an extended version of Young's equation: $\gamma_{s w}=\gamma_{s o}+\gamma_{o w} \cos \theta_{o w}$ In the same way, interfacial tension expressions of $\gamma_{s w}$ and $\gamma_{s o}$ are injected into the extended Young's equation:

$$
\gamma_{o w} \cos \theta_{o w}=\gamma_{w}-\gamma_{o}+2 \sqrt{\gamma_{s}^{d}}\left(\sqrt{\gamma_{o}^{d}}-\sqrt{\gamma_{w}^{d}}\right)+2 \sqrt{\gamma_{s}^{p}}\left(\sqrt{\gamma_{o}^{p}}-\sqrt{\gamma_{w}^{p}}\right)(4)
$$

The liquid-liquid interfacial tension $\gamma_{o w}$ can be either calculated from the same approximation or measured experimentally. Key point from this model is in equation 4: on first approximation, contact angle is sensitive to surface component difference between oil and polar phases $\left(\sqrt{\gamma_{o}^{d / p}}-\sqrt{\gamma_{w}^{d / p}}\right)$ either dispersive or polar. As opposed to the corresponding equation from OWKR's method (eq. 2), this term can 
eventually be neutralized for liquids having similar dispersive component whereas polarity is intrinsically very different between a polar and a non-polar liquid. According to this, contact angle in two liquids has most of the time a stronger dependency on surface polarity than dispersive component. This effect explains qualitatively why two liquids measurement is intrinsically more sensitive to surface polarity when using a polar and a non-polar liquid.

Now similarly to OWKR method, equation 4 leads to a new linearized equation:

$$
\frac{\gamma_{o w} \cos \theta-\gamma_{w}+\gamma_{o}}{2\left(\sqrt{\gamma_{o}^{p}}-\sqrt{\gamma_{w}^{p}}\right)}=\sqrt{\gamma_{s}^{d}} \frac{\left(\sqrt{\gamma_{o}^{d}}-\sqrt{\gamma_{w}^{d}}\right)}{\left(\sqrt{\gamma_{o}^{p}}-\sqrt{\gamma_{w}^{p}}\right)}+\sqrt{\gamma_{s}^{p}}=A X+B
$$

with $X=\frac{\left(\sqrt{\gamma_{o}^{d}}-\sqrt{\gamma_{w}^{d}}\right)}{\left(\sqrt{\gamma_{o}^{p}}-\sqrt{\gamma_{w}^{p}}\right)}$ and $Y=\frac{\gamma_{o w} \cos \theta-\gamma_{w}+\gamma_{o}}{2\left(\sqrt{\gamma_{o}^{p}}-\sqrt{\gamma_{w}^{p}}\right)}$

Dispersive and polar components are now given by the square of slope and y-intercept respectively: $\gamma_{s}^{d}=A^{2}$ and $\gamma_{s}^{p}=B^{2}$ The consequence is that polar component $\gamma_{s}^{p}$ now relates to the $y$-intercept from this equation whereas in the one-liquid method it relates to the slope of the affine function.

The reason for this inversion relates to uncertainty: using a reduced coordinate $Y$ divided by a value close or equal to zero, makes the error diverges, so we deliberately choose to divide by polar components difference. Conversely, in the one liquid method, the dispersive component is the divider, for the exact same reason.

In order to compare latter methods, we used various polar and non-polar reference liquids (table S2 in ESI) to measure contact angles on various low polarity surfaces including Parylene $C$, a high surface energy coating of $\gamma_{s}=35 \mathrm{mN} / \mathrm{m}$ (figure 1 ). We used equation 5 to determine polar and dispersive components from coatings and compared to OWKR method using same coatings or batches of coatings, but measuring one liquid at a time in air. Interfacial tensions between liquids used in the two liquids method are calculated according to equation 2 and given in ESI table S3.
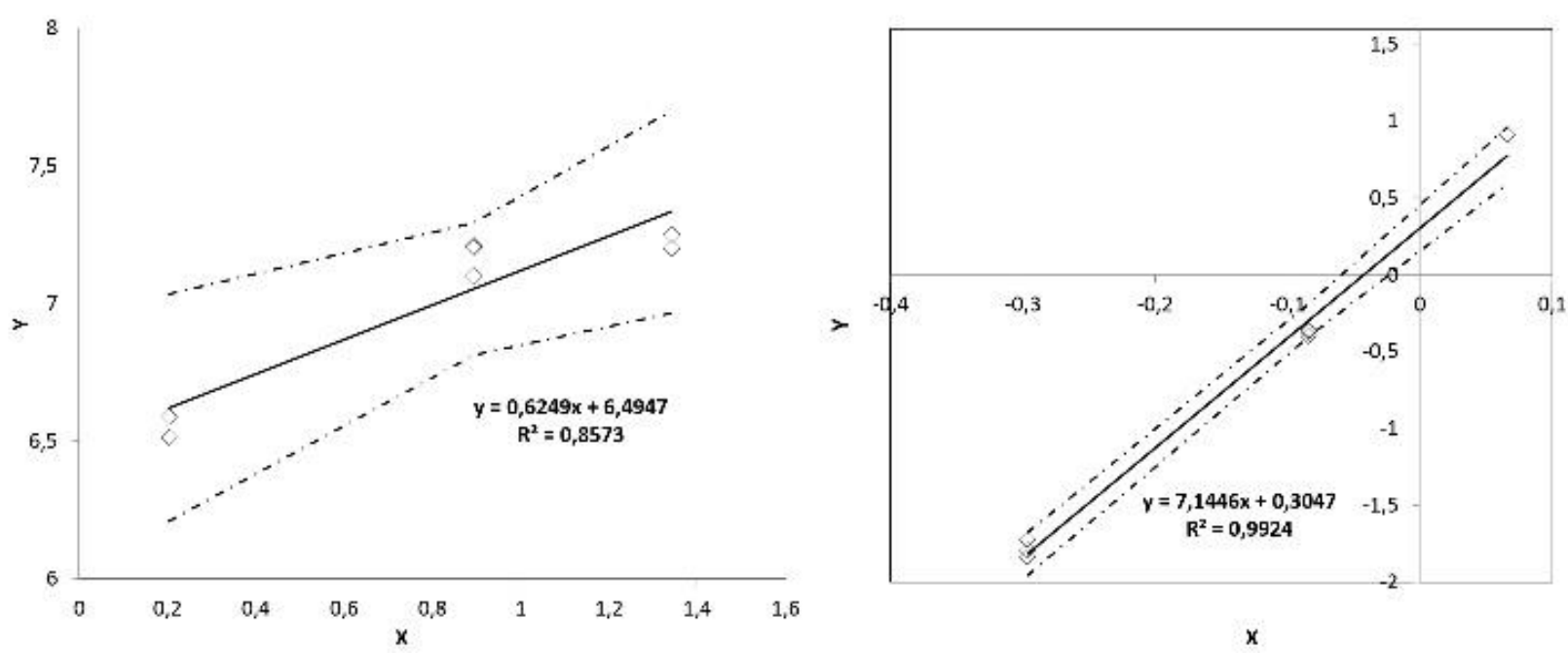

Figure 1: Linearized coordinates $X$ and $Y$ according to equations 3 and 5 measured experimentally (triangles) on Parylene $C$ according to OWKR method (left) and 2-liquids method (right) with liquids indicated in table S2 and interface tension indicated in S3. On both graphs, full line is the linear regression and dashed line is the confidence interval of 95\% according to Student's law.

OWKR's method is very accurate for moderate or relatively high polar surfaces but has an intrinsic limitation for low polarity coatings where a large error is observed in the determination of slope and polar component consequently. 
Based on figure 1, one obvious observation is that we obtain a much better linear estimation of $y$-intercept and slope with a coefficient of regression $r^{2}=0.97$ with 2 -liquids compared to $r^{2}=0.61$ with the OWKR method.

Estimated standard deviation from slope $A$ and $y$-intercept $B$ can be made from a statistical analysis ${ }^{24}: \hat{\sigma}_{\widehat{A}}^{2}=\frac{\widehat{\sigma}_{\varepsilon}^{2}}{\sum\left(x_{i}-\dot{x}\right)^{2}}$ and $\hat{\sigma}_{\widehat{B}}^{2}=$ $\hat{\sigma}_{\varepsilon}^{2}\left[\frac{1}{n}+\frac{\dot{x}^{2}}{\sum\left(x_{i}-\hat{x}\right)^{2}}\right]$ with $\hat{\sigma}_{\varepsilon}^{2}=\frac{\sum_{i}\left(y_{i}-\dot{y}_{i}\right)}{n-2}$ the error variance and $\dot{y}_{i}=\hat{A} x_{i}+\hat{B}$ the estimated values of $y_{i}$ calculated from measured values slope and $\mathrm{y}$-intercept $\hat{A}$ and $\hat{B}$. Estimated values from $\mathrm{A}$ and $\mathrm{B}$ are then expressed with an error of $\mp t\left(1-\frac{\alpha}{2}\right) \hat{\sigma}^{2}$ with $t\left(1-\frac{\alpha}{2}\right)$ the Student's tdistribution law providing uncertainty according to a trust percentage $\alpha$.

Based on this calculation one obtains the results described in figure 1 and table 1 . As can be noted, polar components are much more accurate using the 2-liquids method than the OWKR method.

\begin{tabular}{|l|c|c|c|c|}
\hline \multirow{2}{*}{ Table 1 } & \multicolumn{3}{|c|}{1 liquid } & 2 liquids \\
\cline { 2 - 5 } & $\gamma\left(\mathrm{mJ} / \mathrm{m}^{2}\right)$ & $\gamma^{\mathrm{d}}\left(\mathrm{mJ} / \mathrm{m}^{2}\right)$ & $\gamma^{\mathrm{p}}\left(\mathrm{mJ} / \mathrm{m}^{2}\right)$ & $\gamma^{\mathrm{p}}\left(\mathrm{mJ} / \mathrm{m}^{2}\right)$ \\
\hline Parylene C & $40.8 \pm 0.3$ & $40,1 \pm 0.15$ & $0.7 \pm 0.013$ & $0.1 \pm 0.001$ \\
\hline Fluoropel & $11.30 \pm 0,05$ & $11.1 \pm 0.02$ & $0.3 \pm 0.03$ & $0.01 \pm 0.005$ \\
\hline Cytop & $13.3 \pm 0.07$ & $13.0 \pm 0.03$ & $0.05 \pm 0.03$ & $0.01 \pm 0.003$ \\
\hline SiOC & $23.6 \pm 0.05$ & $23.2 \pm 0.03$ & $0.06 \pm 0.06$ & $0.08 \pm 0.05$ \\
\hline
\end{tabular}

Table 1: Dispersive $\left(\gamma^{d}\right)$ and polar $\left(\gamma^{p}\right)$ surface energy component measured from 1 and 2 liquids methods on various substrate (ESI S4).

In order to understand the origin of this difference, we also calculated intrinsic error from polar and dispersive component from partial derivative $\frac{\partial \gamma_{s}}{\partial \theta}$ on both one and two liquids models for substrate of variable polar and dispersive component:

in the one liquid case we can deduce error from equation 3: $\frac{\partial \gamma_{s}^{p}}{\partial \theta}=-\gamma_{l} \sin \theta \sqrt{\frac{\gamma_{s}^{p}}{\gamma_{l}^{p}}}$ and relative error on surface polar component $\frac{\Delta \gamma_{s}^{p}}{\gamma_{s}^{p}}=$ $\frac{-\gamma_{l}}{\sqrt{\gamma_{s}^{p} \gamma_{l}^{p}}} \sin \theta_{l} \Delta \theta$ and dispersive component $\frac{\Delta \gamma_{s}^{d}}{\gamma_{s}^{d}}=\frac{-\gamma_{l}}{\sqrt{\gamma_{s}^{d} \gamma_{l}^{d}}} \sin \theta_{l} \Delta \theta$

Whereas errors from equation 4 are given by: $\frac{\Delta \gamma_{s}^{p}}{\gamma_{s}^{p}}=\frac{-\gamma_{o w}}{\sqrt{\gamma_{s}^{p}}\left(\sqrt{\gamma_{o}^{p}}-\sqrt{\gamma_{w}^{p}}\right)} \sin \theta_{o w} \Delta \theta$

and $\frac{\Delta \gamma_{s}^{d}}{\gamma_{s}^{d}}=\frac{-\gamma_{o w}}{\sqrt{\gamma_{s}^{d}}\left(\sqrt{\gamma_{o}^{d}}-\sqrt{\gamma_{w}^{d}}\right)} \sin \theta_{o w} \Delta \theta$

Cross calculation on relative error on polar component is not displayed as a function of dispersive component, as it remains constant, similarly to dispersive component error a function of polar component.

Main difference between the 2 methods is illustrated on figure 2: the 2-liquids method is more sensitive to polar component than dispersive interaction, and as a consequence the method exhibits a much larger intrinsic error on dispersive component measurement (Fig. 2A). Inversely, relative error on polar component is intrinsically lower and does not diverge as the OWKR method when polar component tends to zero (Fig. 2B). This particular feature is linked to the fact that, when polar component tends to zero, with one liquid in air, it will necessarily lower contact angle $\theta_{l}$, as overall surface energy increases, whereas using two liquids, contact angle from non-polar liquid will tend to zero. 

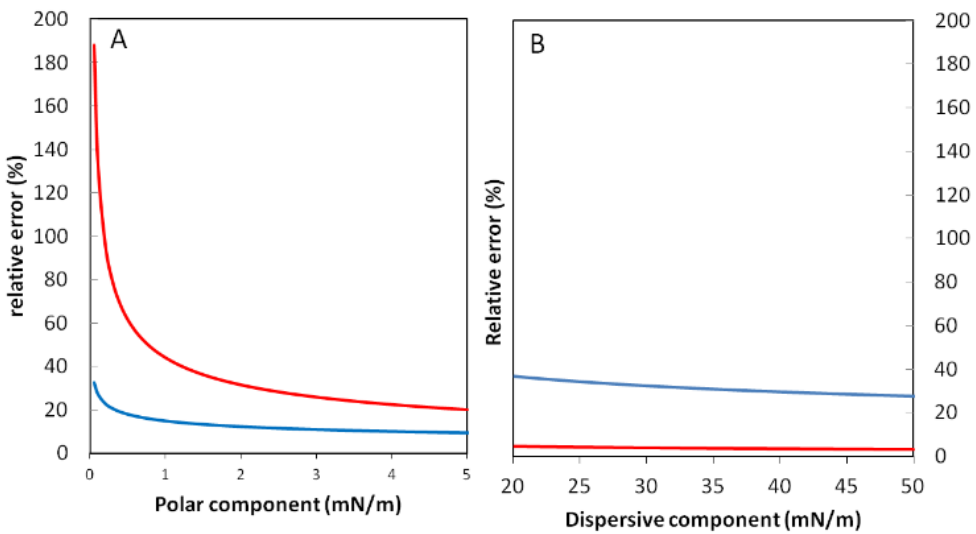

Figure 2: Relative error on polar component calculated on a low surface energy coating $\gamma_{s}=20 \mathrm{mN} / \mathrm{m}$ of variable polarity (A) and relative error on dispersive component on a moderately polar surface $(0.5 \mathrm{mN} / \mathrm{m})$ of variable dispersive component (B). Red line is the calculated error related to the classical OWKR method with water as a reference liquid whereas blue line is the two liquids method with water and dodecane as reference liquids. Contact angle error was estimated from experimental values as $\Delta \theta=0.4 \mathrm{rad}$

In order to validate this new method, we voluntarily performed the same measurements but limiting to three measurements, i.e. water, ethylene glycol and diodomethane for the OWKR method and dodecane, bromonaphtalene and diodomethane in water for the 2 liquids method. This experiment is performed on two low energy fluorinated coatings, Fluoropel and Cytop, and SiOC oxycarbide. It remains obvious from figure 3 that measurement with 2 liquids provides a much more precise measurement of polar component according to regression coefficients and estimated error. When standard method using only three drops of different reference liquids is not accurate enough to estimate polarity from a given surface, two liquids method proves to be largely efficient enough for this kind of determination. 

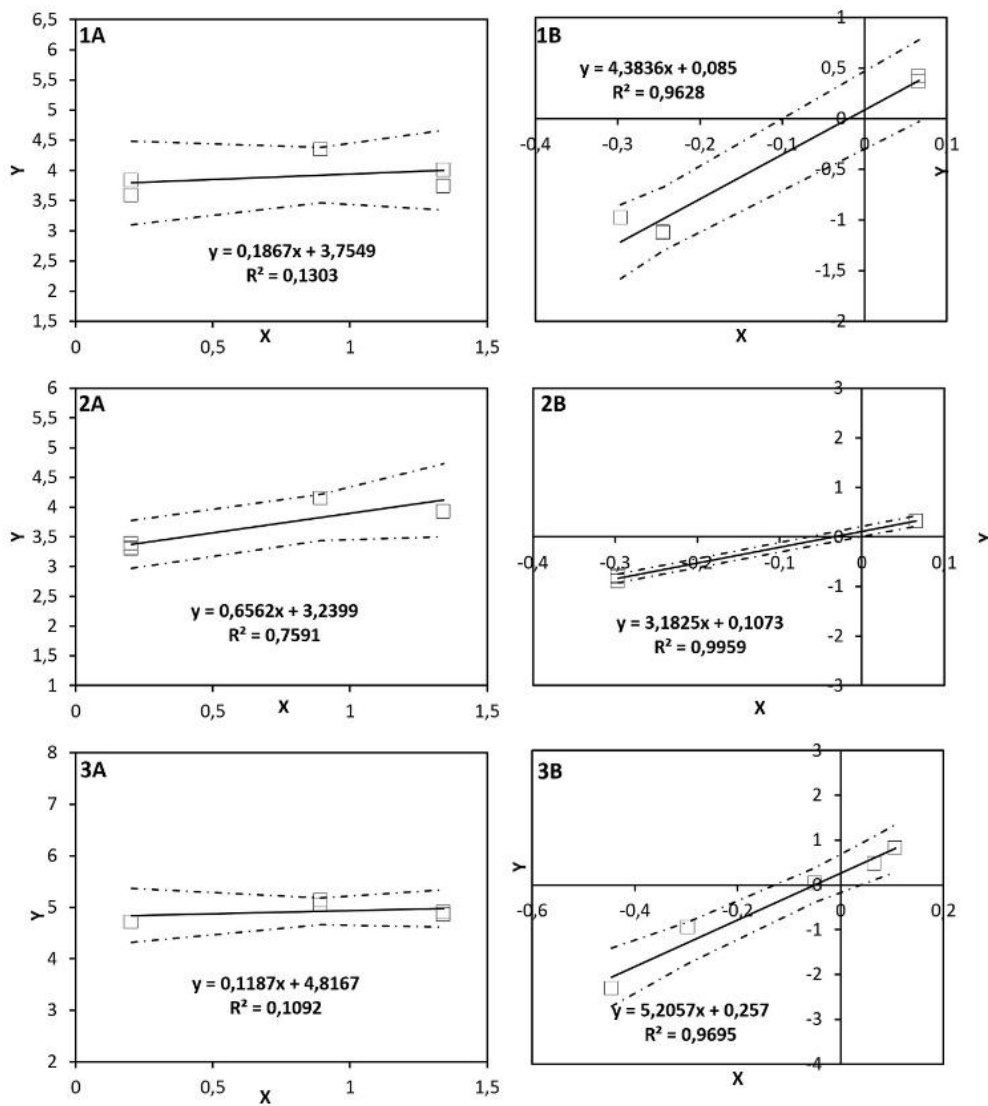

Figure 3: Linearized coordinates measured experimentally (square) on Cytop (1), Fluoropel (2), and oxycarbide SiOC (3) according to the OWKR method (A) and 2-liquids method (B). On both graphs, full line is the linear regression and dashed line is the confidence interval of 95\% according to Student's law. Results have been obtained with only three measurements.

The other huge advantage of this method is to be able to measure surface polarity as a function of $\mathrm{pH}$ in an aqueous environment. In this case contact angle from non-polar liquids is measured in water of variable $\mathrm{pH}$. This of course is not possible when measuring only one liquid, since only water of variable $\mathrm{pH}$ will induce a modification of contact angle. In addition, as expressed by equation 4 , contact angle variation is much more sensitive to polar component in 2-liquids method than in OWKR's. We performed this experiment on Fluoropel and Parylene $\mathrm{C}$ and observed from figure 4 that Parylene $\mathrm{C}$ exhibited a much larger polarity variation with $\mathrm{pH}$. This result is consistent with literature ${ }^{25,26}$ describing parylene as a coating with a negative zeta potential of $-17.5 \mathrm{mV}$ due to the presence of carboxylic groups at the surface of Parylene, related to superficial surface oxidation under oxygen and/or UV light. Our results are also supported by chemical considerations on structure of Parylene C and fluoropel (ESI S5), as Parylene is a halogenated polymer and potentially exhibit surface carboxylic acid group whereas fluoropel only has ester function, that are very weakly hydrolysable, leading to both non polar properties and a better chemical inertia toward $\mathrm{pH}$ variation. Since zeta potential measurement on flat surfaces usually involve electroosmotic current measurement ${ }^{27}$ that are experimentally complex, this method could eventually lead to an easier alternate technic.

This measurement capability can also be used to probe differential affinity of a lipophilic compound in a hydrophilic environment, like proteins or organic compound dispersed in water, as wetting and surface tension are directly linked to adhesion. 


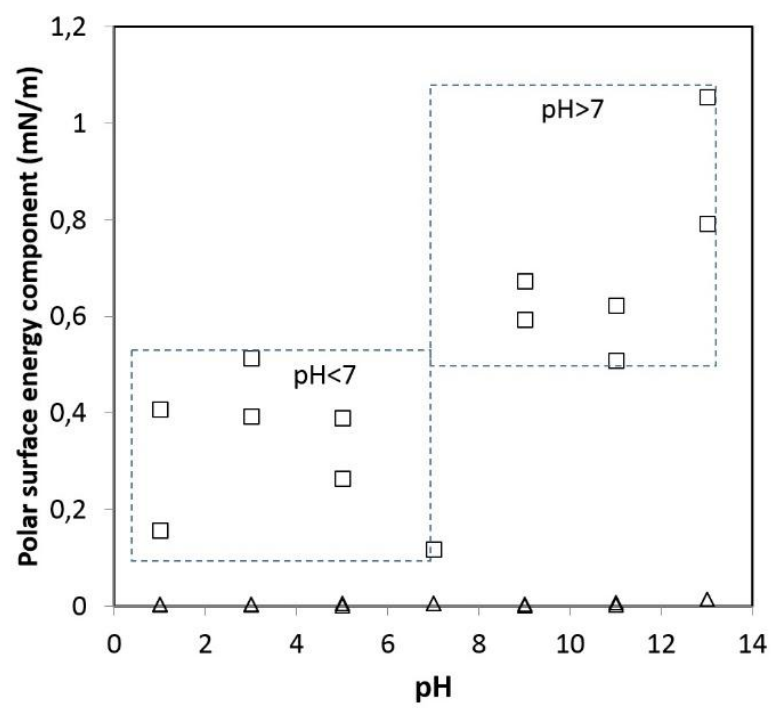

Figure 4: Polar surface energy components on Parylène $C$ (squares) and Fluoropel (triangles) as a function of water pH (measured with diiodomethane, Bromonaphthalene and octane).

\section{Conclusions}

We developed a measurement method based on a two liquids contact angle measurement, allowing much more precise determination of surface polarity, especially on low surface polarity surfaces. This method is also more accurate when performing measurements with a limited number of assays. More importantly, it also allows probing surface properties as a function of pH, measuring surface energy for complex systems where wetting or adhesion is a competition between two compounds varying in lipophilic-hydrophilic nature.

\section{Notes and references}

1 L. A. Girifalco and R. J. Good, J. Phys. Chem., 1957, 61, 904-909.

2 M. Żenkiewicz, J. Achiev. Mater. Manuf. Eng., 2007, 24, 137-145.

3 D. Ge, L. Yang, C. Wang, E. Lee, Y. Zhang and S. Yang, Chem. Commun., 2015, 51, 6149-6152.

B. Luan, T. Huynh and R. Zhou, Nanoscale, 2016, 8, 5750-5754.

5 A. Michiardi, C. Aparicio, B. D. Ratner, J. A. Planell and J. Gil, Biomaterials, 2007, 28, 586-594.

T. Svitova, O. Theodoly, S. Christiano, R. M. Hill and C. J. Radke, Langmuir, 2002, 18, 6821-6829.

7 B. Jalvo, J. Santiago-Morales, P. Romero, R. G. de Villoria and R. Rosal, RSC Adv., 2016, 6, 50278-50287.

T. Young, Philos. Trans. R. Soc. Lond., 1805, 95, 65-87.

9 D. H. Kaelble, J. Adhes., 1970, 2, 66-81.

10 D. K. Owens and R. C. Wendt, J. Appl. Polym. Sci., 1969, 13, 1741-1747.

11 A. Yeung, T. Dabros and J. Masliyah, J. Colloid Interface Sci., 1998, 208, 241-247.

12 E. Chibowski, Adv. Colloid Interface Sci., 2003, 103, 149-172.

13 C. J. van Oss, Colloids Surf. B Biointerfaces, 2007, 54, 2-9.

14 W. A. ZISMAN, in Contact Angle, Wettability, and Adhesion, AMERICAN CHEMICAL SOCIETY, 1964, vol. 43, pp. 1-51.

15 M. N. Bellon-Fontaine, N. Mozes, H. C. van der Mei, J. Sjollema, O. Cerf, P. G. Rouxhet and H. J. Busscher, Cell Biophys., 1990, 17, 93-106.

16 J. J. Jasper, J. Phys. Chem. Ref. Data, 1972, 1, 841-1010.

17 H. J. Busscher, A. W. J. van Pelt, P. de Boer, H. P. de Jong and J. Arends, Colloids Surf., 1984, 9, 319-331.

18 C. Jie-Rong and T. Wakida, J. Appl. Polym. Sci., 1997, 63, 1733-1739.

19 B. Jańczuk and T. Białlopiotrowicz, J. Colloid Interface Sci., 1989, 127, 189-204.

20 A. Kahouli, A. Sylvestre, L. Ortega, F. Jomni, B. Yangui, M. Maillard, B. Berge, J.-C. Robert and J. Legrand, Appl. Phys. Lett., $2009,94,152901$.

21 J. Jakabovič, J. Kováč, M. Weis, D. Haško, R. Srnánek, P. Valent and R. Resel, Microelectron. J., 2009, 40, 595-597.

22 R. Daviau, A. Khan, E. Lisicka-Skrzek, R. N. Tait and P. Berini, Microelectron. Eng., 2010, 87, 1914-1921.

23 M. Maillard, J. Legrand and B. Berge, Langmuir, 2009, 25, 6162-6167.

24 R. Rakotomalala, Stat. Cl. Univ. Lyon, 2015.

25 K. G. Pruden, K. Sinclair and S. Beaudoin, J. Polym. Sci. Part Polym. Chem., 2003, 41, 1486-1496. 
26 T. Goda, T. Konno, M. Takai and K. Ishihara, Colloids Surf. B Biointerfaces, 2007, 54, 67-73.

27 S. L. S. Freire, H. Yang, V. N. Luk and B. O’Brien, Polym.-Plast. Technol. Eng., 2011, 50, 931-936. 